\title{
The existence and uniqueness of solutions to boundary value problems of fractional difference equations
}

\author{
Yuanyuan Pan, Zhenlai Han ${ }^{*}$, Shurong Sun and Zhiqing Huang
}

\begin{abstract}
In this paper, we study the existence and uniqueness of solutions for the boundary value problem $-\Delta_{v-\mu}^{v} y(t)=$ $f(t, y(t+v-1)), \quad \Delta^{j} y(v-N)=0, \quad i \in\{0, \ldots, N-3\}, \Delta^{N-2} y(v-N)=g(y), \Delta_{v-N}^{\mu} y(b+M+v-\mu)=0$, where $v \geq 2,1 \leq \mu<v, f:\{0, \cdots, b+M\} \times \mathbb{R} \rightarrow \mathbb{R}$ is continuous, and nonnegative for $y \geq 0, g: C([v-N, \cdots, b+M+v], \mathbb{R})$ is a given function. We give a representation for the solution to this problem, and we prove the existence and uniqueness of solution to this problem by contraction mapping theorem and Brouwer theorem.
\end{abstract}

Keywords: Discrete fractional equation, Boundary value problem, Existence and uniqueness of solution, Contraction mapping theorem, Brouwer theorem

Mathematics Subject Classification: 2010: 34A08, 34B18

\section{Background}

Gottfried Leibniz and Guilliaume L'Höpital sparked initial curiosity into the theory of fractional calculus during a 1695 correspondence on the possible value and meaning of non-integer-order derivatives. By the late nineteenth century, the combined efforts of a number of mathematicians most notably Liouville, Grünwald, Letnikov, and Riemann produced a fairly solid theory of fractional calculus for functions of a real variable. Though several viable fractional derivatives were proposed, the so-called Riemann-Liouville and Caputo derivatives are the two most commonly used today. Mathematicians have employed this fractional calculus in recent years to model and solve a variety of applied problems. Indeed, as Podlubney outlines in [1], fractional calculus aids significantly in the fields of viscoelasticity, capacitor theory, electrical circuits, electro-analytical chemistry, neurology, diffusion, control theory, and statistics.

The continuous fractional calculus has seen tremendous growth within the last 10 years or so. Some of the recent progress in the continuous fractional calculus has included a paper in which the authors explored

*Correspondence: hanzhenlai@163.com

School of Mathematical Sciences, University of Jinan, Jinan, Shandong 250022, People's Republic of China a continuous fractional boundary value problem of conjugate type, using cone theory, they then deduced the existence of one or more positive solutions [2]. Of particular interest, with regard to the present paper, is the recent work by Benchohra et al. [3]. In that paper, the authors considered a continuous fractional differential equation with nonlocal conditions. Other recent work in the direction of those articles may be found, for example, in [4-14].

A recent interest in discrete fractional calculus has been shown by many scholars, such as Atici and Eloe, who in [15] discuss properties of the generalized falling function, a corresponding power rule for fractional delta-operators and the commutivity of fractional sums. They present in [16] more rules for composing fractional sums and differences. At the same time, a number of papers have appeared, and these have begun to build up the theoretical foundations of the discrete fractional calculus; for example, a recent paper by Atici and Eloe [17] explores some of the theories of a discrete conjugate fractional boundary value problem. Discrete fractional initial value problems were considered in a paper by Atici and Eloe [18] and a paper by the present author [19]. The other recent work may be found in [20-33].

\section{是 Springer}

(C) 2012 Pan et al. licensee Springer. This is an Open Access article distributed under the terms of the Creative Commons Attribution License (http://creativecommons.org/licenses/by/2.0), which permits unrestricted use, distribution, and reproduction in any medium, provided the original work is properly cited. 
Goodrich in [20] considered a discrete fractional boundary value problem of the form

$$
\begin{aligned}
& -\Delta^{v} y(t)=f(t, y(t+v-1)), \\
& y(v-2)=0, \\
& y(v+b)=g(y),
\end{aligned}
$$

where $t \in[0, b]_{\mathbb{N}_{0}}:=\{0,1, \ldots, b\}, f:[v-2, v-1, \ldots, v+$ $b-1]_{\mathbb{N}_{v-2}} \times \mathbb{R} \rightarrow \mathbb{R}$ is a continuous function, $g: C([v-$ $\left.2, v+b]_{\mathbb{N}_{\nu-2}}, \mathbb{R}\right)$ is a given function, and $1<v \leq 2$. He also established the existence and uniqueness of solution to this problem by the contraction mapping theorem, the Brouwer theorem, and the Guo-Krasnosel'kii theorem.

Holm [21] examined the fractional boundary value problem

$$
\begin{cases}-\Delta_{v-\mu}^{v} y(t)=f(t, y(t+v-1)), & \text { for } t \in\{0, \ldots, b+M\}, \\ \Delta^{i} y(\nu-N)=0, & \text { for } i \in\{0, \ldots, N-2\}, \\ \Delta_{v-N}^{\mu} y(b+M+v-\mu)=0, & \end{cases}
$$

where

(1) $v \geq 2$, with $N \in \mathbb{N}$ chosen so that $N-1<v \leq N$.

(2) $1 \leq \mu<v$, with $M \in \mathbb{N}$ chosen so that $M-1<v \leq M$.

(3) $b \in \mathbb{N}$.

(4) $f$ is $\{0,1, \cdots, b+M\} \times \mathbb{R} \rightarrow \mathbb{R}$ is continuous and nonnegative for $y \geq 0$.

Motivated by all the works above, in this paper, we discuss the existence and uniqueness of solutions to a discrete fractional boundary value problem

$$
\left\{\begin{array}{l}
-\Delta_{v-\mu}^{v} y(t)=f(t, y(t+v-1)), \text { for } t \in\{0, \ldots, b+M\} \\
y(v-N)=\cdots=y(v-3)=0, \\
y(v-2)=g(y) \\
\Delta_{v-N}^{\mu} y(b+M+v-\mu)=0,
\end{array}\right.
$$

where

(1) $v \geq 2$, with $N \in \mathbb{N}$ chosen so that $N-1<v \leq N$.

(2) $1 \leq \mu<v$, with $M \in \mathbb{N}$ chosen so that

$$
M-1<v \leq M \text {. }
$$

(3) $b \in \mathbb{N}$.

(4) $f$ is $\{0,1, \cdots, b+M\} \times \mathbb{R} \rightarrow \mathbb{R}$ is continuous and nonnegtive for $y \geq 0$.

(5) $y$ is $\{v-N, \ldots, b+M+v\} \rightarrow \mathbb{R}$.

(6) $g$ is $C([v-N, \ldots, b+M+v], \mathbb{R})$ is a given function.

First, we will give the form of solutions of problem 1 , and then we will prove the existence and uniqueness of solution to this problem by the contraction mapping theorem and the Brouwer theorem. Lastly, we give some examples to illustrate the main results.

\section{Preliminaries}

For the convenience of the reader, we give some background materials from fractional difference theory to facilitate analysis of problem 1 . These and other related results and their proof can be found in [15-18].

Definition 1. We define $t^{\underline{v}}:=\frac{\Gamma(t+1)}{\Gamma(t+1-v)}$, for any $t$ and $v$ for which the right-hand side is defined. We also appeal to the convention that if $t+1-v$ is a pole of the Gamma function and $t+1$ is not a pole, then $t^{\underline{\nu}}=0$.

Definition 2. The vth fractional sum of a function $f$, for $v>0$, is defined by

$$
\Delta^{-v} f(t ; a):=\frac{1}{\Gamma(v)} \sum_{s=a}^{t-v}(t-s-1) \frac{v-1}{-} f(s),
$$

for $t \in\{a+v, a+v+1, \cdots\}=\mathbb{N}_{a+v}$. We also define the $v$ th fractional difference for $v>0$ by $\Delta^{v} f(t)=\Delta^{N} \Delta^{\nu-N} f(t)$, where $t \in \mathbb{N}_{a+v}$ and $v \in \mathbb{N}$ is chosen so that $0 \leq N-1<$ $v \leq N$.

Lemma 1. Let $v$ be any positive real number and $a, b$ be two real numbers such that $v<a \leq b$ [17]. Then the following are valid.

(1) $\frac{1}{x^{\underline{\underline{v}}}}$ is a decreasing function for $x \in(0, \infty)_{\mathbb{N}}$.

(2) $\frac{(a-x)^{\underline{v}}}{(b-x)^{\underline{v}}}$ is a decreasing function for $x \in[0, a-v)_{\mathbb{N}}$.

Lemma 2. If $x<y<t+1$, then $t^{\underline{x}}<t^{\underline{y}}$.

Proof. By definition, we get

$$
\frac{t^{\underline{x}}}{t^{\underline{y}}}=\frac{\frac{\Gamma(t+1)}{\Gamma(t+1-x)}}{\frac{\Gamma(t+1)}{\Gamma(t+1-y)}}=\frac{\Gamma(t+1-y)}{\Gamma(t+1-x)},
$$

in view of $x<y$, then

$$
\frac{\Gamma(t+1-y)}{\Gamma(t+1-x)}<1,
$$

thus

$$
t^{\underline{x}}<t^{\underline{y}}
$$

Lemma 3. Let $f: \mathbb{N}_{a} \rightarrow \mathbb{R}$ and $v>0$ be given with $N-1<v \leq N$ [21]. Consider the vth-order fractional difference equation

$$
\Delta_{a+v-N}^{v} y(t)=f(t), \quad t \in \mathbb{N}_{a}
$$

and the corresponding vth-order fractional initial value problem

$$
\begin{cases}\Delta_{a+v-N}^{v}=f(t), & \text { for } t \in \mathbb{N}_{a}, \\ \Delta^{i} y(a+v-N)=A_{i}, & \text { for } i \in\{0,1, \cdots, N-1\}, A_{i} \in \mathbb{R} .\end{cases}
$$


The general solution to equation 2 is

$$
y(t)=\sum_{i=0}^{N-1} \alpha_{i}(t-a) \frac{i+v-N}{2}+\Delta_{a}^{-v} f(t), \quad t \in \mathbb{N}_{a+v-N}
$$

where $\left\{\alpha_{i}\right\}_{i=0}^{N-1}$ are $N$ real constants. Moreover, the unique solution to Equation 3 is Equation 4 with particular constants

$$
\alpha_{i}=\sum_{p=0}^{i} \sum_{k=0}^{i-p} \frac{(-1)^{k}}{i !}(i-k) \frac{N-v}{i}\left(\begin{array}{c}
i \\
p
\end{array}\right)\left(\begin{array}{c}
i-p \\
k
\end{array}\right) A_{p},
$$

for $i \in\{0,1, \ldots, N-1\}$.

Lemma 4. Let $a \in \mathbb{R}$ and $\mu>0$ be given [21]. Then

$$
\Delta(t-a) \underline{\mu}=\mu(t-a) \stackrel{(\mu-1)}{ },
$$

for any $t$, for which both sides, are well-defined. Furthermore, for $v>0$,

$$
\Delta_{a+\mu}^{-v}(t-a)^{\underline{\mu}}=\mu \frac{-v}{-}(t-a) \stackrel{(\mu+v)}{\underline{n}}, \quad t \in \mathbb{N}_{a+\mu+v}
$$

and

$$
\Delta_{a+\mu}^{v}(t-a) \underline{\mu}=\mu^{-}(t-a) \stackrel{(\mu-v)}{ }, \quad t \in \mathbb{N}_{a+\mu+N-v} .
$$

Lemma 5. Let $f: \mathbb{N}_{a} \rightarrow \mathbb{R}$ be given [21]. For any $v>0$, $\mu>0$ with $M<\mu<M+1$ we have

$$
\begin{aligned}
& \Delta^{v} \Delta_{a}^{-\mu} f(t)=\Delta_{a}^{v-\mu} f(t), \text { for } t \in \mathbb{N}_{a+\mu}, \\
& \Delta^{v} \Delta_{a}^{\mu} f(t)=\Delta_{a}^{v+\mu} f(t), \text { for } t \in \mathbb{N}_{a+M-\mu} .
\end{aligned}
$$

Lemma 6. For $t$ and $s$, for which both $(t-s-1)^{\underline{v}}$ and $(t-s-2)$ - are defined [19], we find that

$$
\Delta_{s}\left[(t-s-1)^{\underline{v}}\right]=-v(t-s-2) \frac{v-1}{\underline{v}} .
$$

Lemma 7. A function $y$ is a solution of problem 1 if and only if $y(t), t \in[v-N, b+M+v]_{\mathbb{N}_{v-N}}$, has the form

$$
\begin{aligned}
y(t)= & \frac{g(y)}{\Gamma(v-1)} t \frac{v-2}{}+\left(\delta-\frac{(v-\mu-1) g(y)}{\Gamma(v)(b+M+2)}\right) t \frac{v-1}{t-v} \\
& -\frac{1}{\Gamma(v)} \sum_{s=0}^{t}(t-\sigma(s)) \frac{v-1}{f}(s, y(s+v-1)),
\end{aligned}
$$

where

$$
\begin{aligned}
\sigma(s) & =s+1, \delta \\
& =\frac{\Gamma(v-\mu) \Delta_{0}^{\mu-v} f(b+M+v-\mu, y(b+M+2 v-\mu-1))}{\Gamma(v)(b+M+v-\mu) \underline{v-\mu-1}} .
\end{aligned}
$$

Proof. From Lemma 3, we get

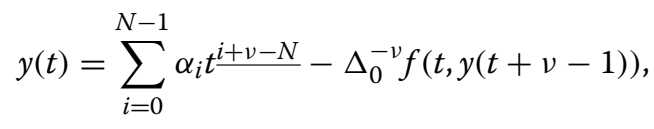

where $\alpha_{i} \in R, \quad t \in[v-N, b+M+v]_{\mathbb{N}_{\nu-N}}$.
By the boundary conditions

$$
y(v-N)=y(v-N+1)=\ldots=y(v-3)=0, y(v-2)=g(y),
$$

we have

$$
\begin{aligned}
& \left.\Delta_{0}^{-v} f(t, y(t+v-1))\right|_{t=v-N+k} \\
& \quad=\left.\left(\frac{1}{\Gamma(v)} \sum_{s=0}^{t-v}(t-\sigma(s)) \frac{v-1}{f} f(s, y(s+v-1))\right)\right|_{t=v-N+k} \\
& \quad=\frac{1}{\Gamma(v)} \sum_{s=0}^{-N+k}(v-N+k-\sigma(s)) \frac{v-1}{} f(s, y(s+v-1)) \\
& =0,
\end{aligned}
$$

for each $k \in\{0,1, \cdots, N-3\}$, we conclude that

$$
\begin{aligned}
0= & y(v-N+k) \\
= & \sum_{i=0}^{N-1} \alpha_{i}(v-N+k) \frac{i+v-N}{} \\
& -\Delta_{0}^{-v} f(v-N+k, y(2 v-N+k-1)) \\
= & \sum_{i=0}^{N-1} \alpha_{i} \frac{\Gamma(v-N+k+1)}{\Gamma(k-i+1)} \\
= & \sum_{i=0}^{k} \alpha_{i} \frac{\Gamma(v-N+k+1)}{(k-i) !} .
\end{aligned}
$$

Solving the system of $N-2$ equations for $\left\{\alpha_{i}\right\}_{i=0}^{N-3}$, we obtain

$$
\alpha_{0}=\alpha_{1}=\cdots=\alpha_{N-3}=0 .
$$

Hence, the general solution to the problem 1 simplifies nicely to

$$
y(t)=\alpha_{N-2} t^{\frac{v-2}{2}}+\alpha_{N-1} t \frac{v-1}{\frac{-v}{-v}} f(t, y(t+v-1)) .
$$

From $y(v-2)=g(y)$,we get

$$
\begin{gathered}
\alpha_{N-2}(v-2) \frac{v-2}{+}+\alpha_{N-1}(v-2) \frac{v-1}{} \\
-\Delta_{0}^{-v} f(v-2, y(2 v-3))=g(y) .
\end{gathered}
$$

Since

$$
\begin{aligned}
& \Delta_{0}^{-v} f(v-2, y(2 v-3)) \\
& \quad=\left.\sum_{s=0}^{t-v}(t-\sigma(s)) \frac{v-1}{f} f(s, y(s+v-1))\right|_{t=v-2}=0
\end{aligned}
$$

then

$$
\Gamma(v-1) \alpha_{N-2}=g(y),
$$

thus, we obtain

$$
\alpha_{N-2}=\frac{g(y)}{\Gamma(v-1)} .
$$


From Lemma 4 and Lemma 5, we get

$$
\begin{aligned}
\Delta_{v-N}^{\mu} y(t)= & \alpha_{N-2} \Delta_{v-N}^{\mu} t \frac{v-2}{\underline{v}}+\alpha_{N-1} \Delta_{v-N}^{\mu} t \frac{v-1}{\nu} \\
& -\Delta_{v-N}^{\mu} \Delta_{0}^{-v} f(t, y(t+v-1)) \\
= & \alpha_{N-2} \Delta_{v-2}^{\mu} t \frac{v-2}{2}+\alpha_{N-1} \Delta_{v-1}^{\mu} t \underline{v-1} \\
& -\Delta_{v}^{\mu} \Delta_{0}^{-v} f(t, y(t+v-1)) \\
= & \alpha_{N-2}(v-2) \underline{\mu} t \underline{v-\mu-2} \\
& +\alpha_{N-1}(v-1) \underline{\mu} t \underline{v-\mu-1} \\
& -\Delta_{v}^{\mu} \Delta_{0}^{-v} f(t, y(t+v-1)) \\
= & \alpha_{N-2} \frac{\Gamma(v-1)}{\Gamma(v-\mu-1)} t \underline{v-\mu-2} \\
& +\alpha_{N-1} \frac{\Gamma(v)}{\Gamma(v-\mu)} t \underline{v-\mu-1} \\
& -\Delta_{0}^{\mu-v} f(t, y(t+v-1)) .
\end{aligned}
$$

In view of

$$
\Delta_{v-N}^{\mu} y(b+M+v-\mu)=0
$$

we get

$$
\begin{aligned}
0= & \Delta_{v-N}^{\mu} y(b+M+v-\mu) \\
= & \alpha_{N-2} \frac{\Gamma(v-1)}{\Gamma(v-\mu-1)}(b+M+v-\mu) \frac{v-\mu-2}{\Gamma(v)} \\
& +\alpha_{N-1} \frac{\Gamma(v-\mu)}{\Gamma(b+M+v-\mu) \frac{v-\mu-1}{}} \\
& -\Delta_{0}^{\mu-v} f(b+M+v-\mu, y(b+M+2 v-\mu-1)),
\end{aligned}
$$

then, we get

$$
\begin{aligned}
& \quad \frac{g(y)}{\Gamma(v-\mu-1)}(b+M+v-\mu) \underline{\nu-\mu-2} \\
& +\alpha_{N-1} \frac{\Gamma(v)}{\Gamma(v-\mu)}(b+M+v-\mu) \underline{v-\mu-1} \\
& -\Delta_{0}^{\mu-v} f(b+M+v-\mu, y(b+M+2 v-\mu-1))=0,
\end{aligned}
$$

thus,

$$
\begin{aligned}
\alpha_{N-1}= & \frac{\Gamma(\nu-\mu) \Delta_{0}^{\mu-v} f(b+M+v-\mu, y(b+M+2 v-\mu-1))}{\Gamma(v)(b+M+v-\mu) \underline{v-\mu-1}} \\
& -\frac{(v-\mu-1) g(y)}{\Gamma(v)(b+M+2)} .
\end{aligned}
$$

Then, we obtain

$$
\begin{aligned}
y(t)= & \frac{g(y)}{\Gamma(v-1)} t^{v-2} \\
& +\left[\frac{\Gamma(v-\mu) \Delta_{0}^{\mu-v} f(b+M+v-\mu, y(b+M+2 v-\mu-1))}{\Gamma(v)(b+M+v-\mu) \frac{v-\mu-1}{}}\right. \\
& \left.-\frac{(v-\mu-1) g(y)}{\Gamma(v)(b+M+2)}\right] t^{\nu-1}-\Delta_{0}^{-v} f(t, y(t+v-1)) .
\end{aligned}
$$

Let

$\delta=\frac{\Gamma(v-\mu) \Delta_{0}^{\mu-v} f(b+M+v-\mu, y(b+M+2 v \mid !-\mu-1))}{\Gamma(v)(b+M+v-\mu) \underline{v-\mu-1}}$.
We get

$$
\begin{aligned}
y(t)= & \frac{g(y)}{\Gamma(v-1)} t^{\nu-2}+\left(\delta-\frac{(v-\mu-1) g(y)}{\Gamma(\nu)(b+M+2)}\right) t^{\nu-1} \\
& -\frac{1}{\Gamma(v)} \sum_{s=0}^{t-v}(t-\sigma(s)) \frac{v-1}{f} f(s, y(s+v-1)) .
\end{aligned}
$$

This show that if problem 1 has a solution, then it can be represented by Equation 6 and that every function of form Equation 6 is a solution of problem 1, which completes the proof.

\section{Results and discussion}

In this section, we wish to show that under certain conditions, problem 1 have at least one solution. We notice that problem 1 may be recast an equivalent summation equation. In particular, $y$ is a solution of problem 1 if and only if $y$ is a fixed point of the operator $T: \mathbb{R}^{b+M+N+1} \rightarrow$ $\mathbb{R}^{b+M+N+1}$, where

$$
\begin{aligned}
& (T y)(t):=\frac{t \underline{v-2}}{\Gamma(v-1)} g(y)+\delta t \frac{\nu-1}{-}-\frac{(v-\mu-1) t \frac{\nu-1}{}}{\Gamma(\nu)(b+M+2)} g(y) \\
& -\frac{1}{\Gamma(v)} \sum_{s=0}^{t-v}(t-\sigma(s)) \frac{v-1}{f} f(s, y(s+v-1)),
\end{aligned}
$$

for $t \in[v-N, b+M+v]_{\mathbb{N}_{\nu-N}}$, where this observation follows from Lemma 7. We now use this fact to prove our first existence theorem.

Theorem 1. Define $\|y\|=\sup _{t \in[v-N, b+M+v]_{\mathbb{N}_{\nu-N}}} y(t)$. Suppose that $f(t, y)$ and $g(y)$ are Lipschitz in $y$. That is, there exist $\alpha, \beta>0$ such that $\left|f\left(t, y_{1}\right)-f\left(t, y_{2}\right)\right| \leq \alpha \mid y_{1}-$ $y_{2}|| g,\left(y_{1}\right)-g\left(y_{2}\right)|\leq \beta| y_{1}-y_{2} \mid$ whenever $y_{1}, y_{2} \in R$. Then it follows that problem 1 has a unique solution provided that the condition

$$
\begin{aligned}
0<\alpha \prod_{j=1}^{b+M}\left(\frac{v+j}{j}\right) & +\beta \frac{(b+M+v) \frac{v-2}{\Gamma(v-1)}}{\Gamma(\nu)(b+M+2)} \\
& +\beta \frac{(v-\mu-1)(b+M+v) \frac{v-1}{2}}{\beta}<1
\end{aligned}
$$

holds.

Proof. We will show that $T$ is a contraction mapping. To this end, we notice that for given $y_{1}$ and $y_{2}$, 


$$
\begin{gathered}
\left\|T y_{1}-T y_{2}\right\| \leq \beta\left\|y_{1}-y_{2}\right\| \max _{t \in[v-N, b+M+v]}\left[\frac{t \frac{v-2}{\Gamma(\nu-1)}}{\Gamma(v-1)}\right] \\
+\beta\left\|y_{1}-y_{2}\right\| \max _{t \in[v-N, b+M+v]}\left[\frac{\left.(v-\mu-1) t \frac{\nu-1}{\Gamma(v)(b+M+2)}\right]}{+\alpha\left\|y_{1}-y_{2}\right\|} \max _{t \in[v-N, b+M+v]}\left[\frac{1}{\Gamma(v)} \sum_{s=0}^{t-v}(t-\sigma(s))^{\nu-1}\right] .\right.
\end{gathered}
$$

We now analyze each of the three terms on the righthand side of proof 9 .

Firstly, by an application of Lemma 6, we get

$$
\begin{aligned}
\frac{1}{\Gamma(v)} \sum_{s=0}^{t-v}(t-\sigma(s))^{v-1} & =\frac{1}{\Gamma(v)}\left[-\frac{1}{v}(t-s)^{\underline{v}}\right]_{s=0}^{t-v+1} \\
& =\frac{\Gamma(t+1)}{\Gamma(v+1) \Gamma(t+1-v)} \\
& \leq \frac{\Gamma(b+M+v+1)}{\Gamma(b+M+1) \Gamma(v+1)} \\
& =\prod_{j=1}^{b+M}\left(\frac{v+j}{j}\right) .
\end{aligned}
$$

Secondly, by Lemma 1,

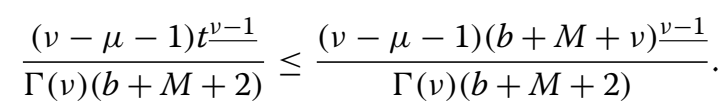

Finally, from another application of Lemma 1, we obtain

$$
\frac{t^{\nu-2}}{\Gamma(v-1)} \leq \frac{(b+M+v) \underline{v-2}}{\Gamma(v-1)} .
$$

So, putting inequalities 10,11,12 in Equation 9, we conclude that

$$
\begin{aligned}
\left\|T y_{1}-T y_{2}\right\| \leq & \left\{\alpha \prod_{j=1}^{b+M}\left(\frac{v+j}{j}\right)+\beta \frac{(b+M+v) \frac{v-2}{\Gamma(v-1)}}{\Gamma(v)(b+M+2)}\right\}\left\|y_{1}-y_{2}\right\| .
\end{aligned}
$$

Therefore, by requiring condition 8 to hold, we find that problem 1 has a unique solution. And this completes the proof.

By weakening the conditions imposed on $f(t, y)$ and $g(y)$, we can still deduce the existence of a solution to problem 1 . We will apply the Brouwer theorem to accomplish this.
Theorem 2. Suppose that there exists a constant $K>$ $2 \delta(b+M+v) \frac{v-1}{2}$ such that $f(t, y)$ satisfies the inequality

$$
\max _{(t, y) \in[0, b+M]_{\mathbb{N}_{0}} \times[-K, K]}|f(t, y)| \leq \frac{K-\delta(b+M+v) \frac{\nu-1}{m_{1}+m_{2}+m_{3}}}{m}
$$

and $g(y)$ satisfies the inequality

$$
\max _{y \in[-K, K]}|g(y)| \leq \frac{K-\delta(b+M+v) \frac{v-1}{}}{m_{1}+m_{2}+m_{3}},
$$

where $m_{1}=\frac{1}{\Gamma(v)} \sum_{s=0}^{b+M}(b+M+v-\sigma(s)) \stackrel{v-1}{2}, m_{2}=$ $\frac{(b+M+v) \underline{v-2}}{\Gamma(\nu-1)}, m_{3}=\frac{|v-\mu-1|(b+M+v) \underline{v-2}}{\Gamma(v)}, \delta$ is defined as in 5.

Then problem 1 has at least one solution, $y_{0}$, satisfying $\left|y_{0}\right| \leq K$, for all $t \in[v-N, b+M+v]_{\mathbb{N}_{v-N}}$.

Proof. Consider the Banach space $\mathscr{B}:= \begin{cases}y \in \\ \text { e }\end{cases}$ $\left.\mathbb{R}^{b+M+N+1}:\|y\| \leq K\right\}$. Let $T$ be the operator defined in Equation 7. It is clear that $T$ is a continuous operator. Therefore, the main objective in establishing this result is to show that $T: \mathscr{B} \rightarrow \mathscr{B}$. That is, whenever $\|y\| \leq K$, it follows that $\|T y\| \leq K$. Once this is established, the Brouwer theorem will be invoked to deduce the conclusion.

To this end, assume that inequalities in 13 and 14 hold for given $f$ and $g$. For convenience, we let

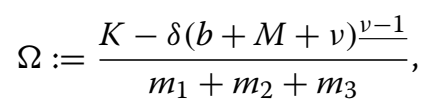

which is a strictly positive constant. Using the notation introduced in 15 , we have

$$
\begin{aligned}
& \|T y\| \leq \max _{t \in[\nu-N, b+M+\nu]_{\mathbb{N}-N}} \frac{t^{\frac{\nu-2}{}}}{\Gamma(v-1)}|g(y)| \\
& +\delta \max _{t \in[\nu-N, b+M+\nu]_{\mathbb{N}_{\nu}-N}} t \underline{\nu-1}
\end{aligned}
$$

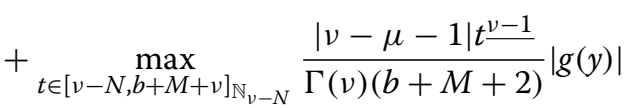

$$
\begin{aligned}
& +\max _{t \in[v-N, b+M+v]_{\mathbb{N}_{v}-N}} \\
& \times \frac{1}{\Gamma(v)} \sum_{s=0}^{t-v}(t-\sigma(s))^{\frac{v-1}{2}}|f(t, y(s+v-1))|
\end{aligned}
$$

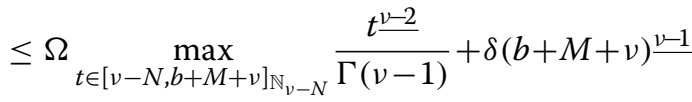

$$
\begin{aligned}
& +\Omega \max _{t \in[v-N, b+M+v]_{\mathbb{N}_{\nu-N}}} \frac{|v-\mu-1| t \underline{\nu-1}}{\Gamma(v)(b+M+2)} \\
& +\Omega_{t \in[v-N, b+M+v]_{\mathbb{N}_{v-N}}} \frac{1}{\Gamma(v)} \sum_{s=0}^{t-v}(t-\sigma(s))^{\frac{\nu-1}{2}} .
\end{aligned}
$$


Now, much as in the proof of Theorem 1, we can simplify the expression on the right-hand side of inequality 16 . Indeed, we know that

$$
\frac{1}{\Gamma(v)} \sum_{s=0}^{t-v}(t-\sigma(s)) \frac{v-1}{\Gamma(v)} \sum_{s=0}^{b+M}(b+M+v-\sigma(s)) \frac{v-1}{=}=m_{1},
$$

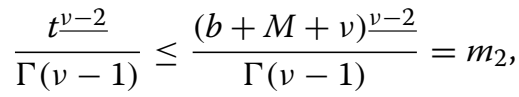

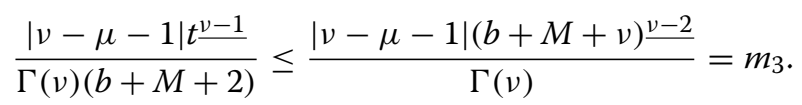

We now put inequalities in 18,19,20 together, we get

$$
\|T y\| \leq \Omega\left(m_{1}+m_{2}+m_{3}\right)+\delta(b+M+v) \frac{v-1}{\leq} \leq .
$$

Thus, from Equation 20 we deduce that $T: \mathscr{B} \rightarrow \mathscr{B}$, as desired. Consequently, it follows at once by the Brouwer theorem that there exists a fixed point of the map $T$, say $T y_{0}=y_{0}$, with $y_{0} \in \mathscr{B}$. So this function $y_{0}$ is a solution of problem 1. Moreover, $y_{0}$ satisfies the bound $\left|y_{0}(t)\right| \leq K$ for each $t \in[v-N, b+M+$ $\nu]_{\mathbb{N}_{\nu-N}}$. Thereby, this completes the proof of the theorem.

\section{Example}

In this section, we will present some examples to illustrate the main results.

Example 1. Suppose that $v=\frac{7}{3}, b=1, M=2, \mu=\frac{5}{4}$ and $N=3$. Let $f(t, y):=\frac{\sin y}{50+|t|}$ and $g(y):=\frac{1}{50} \cos y$. Then problem 1 becomes

$$
\left\{\begin{array}{l}
-\Delta_{\frac{13}{12}}^{\frac{7}{3}} y(t)=\frac{\sin \left(y\left(t+\frac{4}{3}\right)\right)}{50+\left|t+\frac{4}{3}\right|}, \text { for } \quad t \in\{0, \ldots, 3\} \\
y\left(-\frac{2}{3}\right)=0 \\
\Delta y\left(-\frac{2}{3}\right)=\frac{1}{50} \cos \left(y\left(t+\frac{4}{3}\right)\right) \\
\Delta_{-\frac{2}{3}}^{\frac{5}{4}} y\left(\frac{49}{12}\right)=0 .
\end{array}\right.
$$

In this case, inequality 8 is

$$
\begin{aligned}
0< & \alpha \prod_{j=1}^{b+M}\left(\frac{v+j}{j}\right)+\beta \frac{(b+M+v) \frac{v-2}{\Gamma(v-1)}}{\Gamma(v)(b+M+2)} \\
& +\beta \frac{(v-\mu-1)(b+M+v) \frac{v-1}{3}}{=} \\
= & \prod_{j=1}^{3}\left(\frac{\frac{7}{3}+j}{j}\right)+\beta \frac{\frac{16}{3} \frac{1}{3}}{\Gamma\left(\frac{4}{3}\right)}+\beta \frac{\frac{1}{12} \times \frac{16}{3} \frac{4}{3}}{\Gamma\left(\frac{7}{3}\right) \times 5} \\
< & 13 \alpha+\frac{39}{2} \beta .
\end{aligned}
$$

In addition, $f$ and $g$ are with Lipschitz constant $\alpha=\beta=$ $\frac{1}{50}$. So, for these choices $\alpha$ and $\beta$, problem 22 is satisfied. Therefore, from Theorem 1 we deduce that problem 21 has a unique solution.

Example 2. Suppose that $v=\frac{9}{4}, b=8, M=2, \mu=\frac{5}{4}$, $N=3$ and $K=1,000$. Let $f(t, y):=\frac{1}{100} t \exp \left\{-\frac{1}{100} y^{2}\right\}$ and $g(y):=\sum_{i=1}^{n} c_{i} y\left(t_{i}\right), t_{i} \in[v-N, v+b+M]_{\mathbb{N}_{v-N}}$. Then problem 1 is

$$
\left\{\begin{array}{l}
-\Delta_{1}^{\frac{9}{4}} y(t)=\frac{1}{100} t \exp \left\{-\frac{1}{100} y^{2}\left(t+\frac{5}{4}\right)\right\}, \text { for } t \in\{0, \cdots, 3\} \\
y\left(-\frac{3}{4}\right)=0 \\
\Delta y\left(-\frac{3}{4}\right)=\sum_{i=1}^{n} c_{i} y\left(t_{i}+\frac{5}{4}\right) \\
\Delta_{-\frac{3}{4}}^{\frac{5}{4}} y(11)=0
\end{array}\right.
$$

so, the Banach space $\mathscr{B}:=\left\{y \in \mathbb{R}^{14}:\|y\| \leq 1,000\right\}$.

For

$$
\begin{aligned}
& m_{1}=\frac{1}{\Gamma\left(\frac{9}{4}\right)} \sum_{s=0}^{10}\left(10+\frac{9}{4}-\sigma(s)\right)^{\frac{5}{4}} \leq 60, \\
& m_{2}=\frac{\left(10+\Gamma\left(\frac{9}{4}\right)^{\frac{1}{4}}\right.}{\Gamma\left(\frac{5}{4}\right)} \leq 11, \quad m_{3}=0, \\
& \delta(b+M+v)^{\frac{v-1}{4}} \leq 500,
\end{aligned}
$$

thus

$$
\frac{K-\delta(b+M+v) \frac{v-1}{2}}{m_{1}+m_{2}+m_{3}} \geq \frac{500}{60+11} \geq \frac{25}{4} .
$$

It is clear that $f(t, y) \leq \frac{49}{400}<\frac{25}{4}$. And if we require

$$
\sum_{i=1}^{n}\left|c_{i}\right| \leq \frac{1}{10000},
$$


then from 25, for $y \in \mathscr{B}, g(y) \leq \frac{1}{10}<\frac{25}{4}$ so that $f$ and $g$ satisfy the conditions. Thus, by Theorem 2 , we deduce that problem 23 has at least one solutions.

\section{Endnotes}

This research is supported by the Natural Science Foundation of China (11071143, 60904024, 61174217), Natural Science Outstanding Youth Foundation of Shandong Province (JQ201119) and supported by Shandong Provincial Natural Science Foundation (ZR2010AL002, ZR2009AL003), also supported by Natural Science Foundation of Educational Department of Shandong Province (J11LA01).

\section{Acknowledgements}

The authors sincerely thank the reviewers for their valuable suggestions and useful comments that have lead to the present improved version of the original manuscript.

Received: 9 April 2012 Accepted: 11 June 2012

Published: 11 June 2012

\section{References}

1. Podlubny, I: Fractional differential equations, mathematics in science and engineering. Academic Press, New York/London/Toronto (1999)

2. Bai, Z, Lü, H: Positive solutions for boundary value problem of nonlinear fractional differential equation. J. Math. Anal. Appl. 311, 495-505 (2005)

3. Benchohra, M, Hamani, S, Ntouyas, SK: Boundary value problems for differential equations with fractional order and nonlocal conditions. Nonlinear Anal. TMA. 71, 2391-2396 (2009)

4. Diethelm, K, Ford, N: Analysis of fractional differential equations. J. Math. Anal. Appl. 265, 229-248 (2002)

5. $\mathrm{Xu}, \mathrm{X}$, Jiang, D, Yuan, C: Multiple positive solutions for the boundary value problem of a nonlinear fractional differential equation. Nonlinear Anal. TMA. 71, 4676-4688 (2009)

6. Zhao, Y, Sun, S, Han, Z, Li, Q: The existence of multiple positive solutions for boundary value problems of nonlinear fractional differential equations. Comm. Nonlinear Sci. Numer. Simul. 16, 2086-2097 (2011)

7. Zhao, Y, Sun, S, Han, Z, Li, Q: Positive solutions to boundary value problems of nonlinear fractional differential equations. Abstr. Appl. Anal. 2011, 1-16 (2011)

8. Zhao, Y, Sun, S, Han, Z, Li, Q: Theory of fractional hybrid differential equations. Comput. Math. Appl. 62, 1312-1324 (2011)

9. Zhao, Y, Sun, S, Han, Z, Zhang, M: Positive solutions for boundary value problems of nonlinear fractional differential equations. Appl. Math. Comput. 217, 6950-6958 (2011)

10. Zhao, Y, Sun, S, Han, Z, Feng, W: Positive solutions for a coupled system of nonlinear differential equations of mixed fractional orders. Adv. Differ. Equ. 10, 1-13 (2011)

11. Zhou, $Y$, Jiao, $F, L i$, J: Existence and uniqueness for p-type fractional neutral differential equations. Nonlinear Anal. 71(7-8), 2724-2733 (2009)

12. Zhou, Y, Jiao, F, Li, J: Existence and uniqueness for fractional neutral differential equations with infinite delay. Nonlinear Anal. 71(7-8), 3249-3256 (2009)

13. Zhou, Y, Jiao, F: Nonlocal cauchy problem for fractional evolution equations. Nonlinear Anal. 11, 4465-4475 (2010)

14. Bastos, NRO, Mozyrska, D, Torres, DFM: Fractional derivatives and integrals on time scales via the inverse generalized Laplace transform. Int. J. Math. Comput. 11, 1-9 (2011)

15. Atici, FM, Eloe, FM: A transform method in discrete fractional calculus. Int. J. Difference Equ. 2, 165-176 (2007)

16. Atici, FM, Sengül, S: Modeling with fractional difference equations. J. Math. Anal. Appl. 369, 1-9 (2010)

17. Atici, FM, Eloe, PW: Two-point boundary value problems for finite fractional difference equations. J. Difference Equ. Appl. 17, 445-456 (2011)

18. Atici, FM, Eloe, PW: Initial value problems in discrete fractional calculus. Proc. Amer. Math. Soc. 137(3), 981-989 (2009)
19. Goodrich, CS: Continuity of solutions to discrete fractional initial value problems. Comput. Math. Appl. 59, 3489-3499 (2010)

20. Goodrich, CS: Existence and uniqueness of solutions to a fractional difference equation with nonlocal conditions. Comput. Math. Appl. 61, 191-202 (2011)

21. Holm, MT: The Theory of Discrete Fractional Calculus: Development and Application. University of Nebraska-Lincoln, Nebraska (2011)

22. Agarwal, RP, Meehan, M, ORegan, D: Fixed Point Theory and Applications. Cambridge University Press, Cambridge, UK (2001)

23. Pan, Y, Han, Z: Existence of solutions for a coupled system of boundary value problem of nonlinear fractional differential equations. In: Proceedings of the 5 th International Congress on Mathematical Biology, Nanjing University of Information Science \& Technology, Nanjing City, 3-5 June 2011. 1 (2011)

24. Goodrich, CS: Solutions to a discrete right-focal fractional boundary value problem. Int. J. Difference Equ. 5, 195-216 (2010)

25. Goodrich, CS: Existence of a positive solution to a system of discrete fractional boundary value problems. Appl. Math. Comput. 217, 4740-4753 (2011)

26. Henderson, J, Ntouyas, SK, Purnaras, IK: Positive solutions for systems of nonlinear discrete boundary value problems. J. Difference Equ. Appl. 15 895-912 (2009)

27. Dunninger, DR, Wang, H: Existence and multiplicity of positive solutions for elliptic systems. Nonlinear Anal. 29, 1051-1060 (1997)

28. Goodrich, CS: On positive solutions to nonlocal fractional and integer-order difference equations. Appl. Anal. Discrete Math. 5, 122-132 (2011)

29. Goodrich, CS: On discrete sequential fractional boundary value problems. J. Math. Anal. Appl. 385, 111-124 (2012)

30. Ferreira, RAC: Positive solutions for a class of boundary value problems with fractional q-differences. Comput. Math. Appl. 61, 367-373 (2011)

31. Bastos, NRO, Ferreira, RAC, Torres, DFM: Discrete-time fractional variational problems. Signal Processing. 91, 513-524 (2011)

32. Pan, Y, Han, Z, Sun, S, Zhao, Y: The existence of solutions to a system of discrete fractional boundary value problems. Abstr. Appl. Anal. 2012, $1-15$ (2012)

33. Goodrich, CS: Existence of a positive solution to a class of fractional differential equations. Appl. Math. Lett. 23, 1050-1055 (2010)

doi:10.1186/2251-7456-6-7

Cite this article as: Pan et al.: The existence and uniqueness of solutions to boundary value problems of fractional difference equations. Mathematical Sciences 2012 6:7.

\section{Submit your manuscript to a SpringerOpen ${ }^{\circ}$ journal and benefit from:}

- Convenient online submission

- Rigorous peer review

- Immediate publication on acceptance

- Open access: articles freely available online

- High visibility within the field

- Retaining the copyright to your article

Submit your next manuscript at $\boldsymbol{\nabla}$ springeropen.com 